\title{
A graph theory based new approach for power system restoration
}

DOI:

10.1109/PTC.2013.6652108

Link to publication record in Manchester Research Explorer

\section{Citation for published version (APA):}

Quiros-Tortos, J., \& Terzija, V. (2013). A graph theory based new approach for power system restoration. In 2013 IEEE Grenoble Conference PowerTech, POWERTECH 2013/IEEE Grenoble Conf. PowerTech, POWERTECH IEEE. https://doi.org/10.1109/PTC.2013.6652108

\section{Published in:}

2013 IEEE Grenoble Conference PowerTech, POWERTECH 2013|IEEE Grenoble Conf. PowerTech, POWERTECH

\section{Citing this paper}

Please note that where the full-text provided on Manchester Research Explorer is the Author Accepted Manuscript or Proof version this may differ from the final Published version. If citing, it is advised that you check and use the publisher's definitive version.

\section{General rights}

Copyright and moral rights for the publications made accessible in the Research Explorer are retained by the authors and/or other copyright owners and it is a condition of accessing publications that users recognise and abide by the legal requirements associated with these rights.

\section{Takedown policy}

If you believe that this document breaches copyright please refer to the University of Manchester's Takedown Procedures [http://man.ac.uk/04Y6Bo] or contact uml.scholarlycommunications@manchester.ac.uk providing relevant details, so we can investigate your claim.

\section{OPEN ACCESS}




\section{A Graph Theory Based New Approach for Power System Restoration}

\author{
Jairo Quirós-Tortós, Student Member, IEEE \\ The University of Manchester \\ School of Electrical \& Electronic Engineering \\ Electrical Energy and Power Systems \\ Manchester, UK \\ jairoquirostortos@ieee.org
}

\begin{abstract}
This paper presents a novel graph theory based approach for restoring large scale power systems affected by complete blackouts. The proposed graph theory based method assesses the power system in blackout and represents the required information in a graph. The graph is initially used to determine the sectionalizing strategy. To do so, an un-normalized spectral clustering algorithm is implemented. The method then applies parallel power system restoration, restoring the created islands in parallel. Thus, the restoration process is speeded up. An algorithm for computing the $k$-shortest simple paths from the blackstart unit in each island to the non-blackstart units and loads in the same island is then carried out. By implementing the proposed method, cranking power can be rapidly sent to nonblackstart units and loads can be picked up quickly. The new graph theory based method is tested on the New England 39-bus test system. Simulation results are used to demonstrate the effectiveness of the proposed method on restoring systems affected by complete blackouts.
\end{abstract}

Index Terms-Graph theory, parallel power system restoration, spectral clustering.

\section{INTRODUCTION}

Power System Restoration (PSR) is the process carried out after a partial or complete power system blackout. Restoring the network as quickly as possible, taking into consideration the static and dynamic constraints previously defined by the grid codes, is the main objective of system operators during PSR. By speeding up the restoration process, system operators directly reduce the cost of the power system blackout.

Two main strategies to restore power systems are stated in [1] and [2]. Nevertheless, it should be mentioned that the selection of the strategy to be implemented in a particular power system depends mainly on the characteristics of that network. The "build-up" approach sectionalizes the entire power system into smaller subsystems, also called islands. Then, the created islands are restored in parallel by applying Parallel Power System Restoration (PPSR). On the other hand, the "build-down" strategy reenergizes the bulk network before resynchronizing loads and generators. Extended analysis of these two strategies is presented in [1]-[5].

This work was supported in part by the Engineering and Physical Science Research Council (EPSRC) in the UK and The University of Costa Rica.

\author{
Vladimir Terzija, Senior Member, IEEE \\ The University of Manchester \\ School of Electrical \& Electronic Engineering \\ Electrical Energy and Power Systems \\ Manchester, UK \\ terzija@ieee.org
}

Since the restoration duration is significantly reduced when implementing the "build-up" strategy, PPSR is considered as the predominantly used worldwide restoration scheme. When applying the "build-up" strategy, the power system affected by the blackout is assessed first. Then, the Sectionalizing Strategy (SS) is determined. The SS must define the boundary of the islands, such that each island has at least one Blackstart (BS) unit [1]-[6]. Other important constraints to be considered when determining the SS are mentioned in Section II. When the SS is identified, the islands are restored in parallel. When the created islands are completely stable, these are resynchronized and the entire system is finally restored. During the synchronization of the created islands, the monitoring of the boundary buses is vital for a successful restoration.

Two main advantages on applying PPSR are: (i) the restoration time duration is considerably reduced, and (ii) in case of a large disturbance in an island, a recurrent blackout would only occur in the affected area [1]-[3]. However, as the network is separated into islands, the stability margins of the islands are an important issue to be considered during PPSR. Also, different blackstart (BS) units are required to be available and distributed in the network.

Research on determining the optimal unit restarting sequence and different network reconfiguration approaches can be found in [6]-[8]. These methods present interesting results for these two particular problems during the restoration process. However, more general methods to aid operators during the restoration process are still required.

As PPSR presents several advantages over the "builddown" strategy, this paper presents a new graph theory based method to restore large scale power systems affected by complete blackouts. By representing the network as an undirected graph, the first step of the proposed method separates the affected network into the fewest islands required to satisfy various PPSR constraints [1]-[6]. This first step considers information obtained from the assessment of the network in blackout. Unavailable machines, lines without synchro-check relays, and information from system operators are considered and eventually used when computing the SS. This SS is computed using an un-normalized spectral clustering (USC) algo- 
rithm. The determined SS must create islanding satisfying BS availability, providing sufficient power generation within each island and ensuring that the tie-lines are equipped with synchro-check relays

The second step, which is carried out simultaneously for each of the created islands, applies an efficient algorithm to determine the $k$-shortest simple paths between the BS unit in each island to the non-blackstart (NBS) units and loads in the same island. When computing the shortest paths between any two elements, the number of connections required (number of edges) and the electrical distance between these elements are determined. Then, the determined paths are ranked based on the number of connections required for energizing the element and the electrical distance between these. It must be noted that the number of connections required is proportional to the time duration to restore that element. As Critical loads (CLs) are more important than common loads, these are picked up first. In addition, cranking power is rapidly sent to NBS units. Finally, the sequence of events for each island is presented and the estimated time to restore the elements is determined. It is important to mention that this paper does not consider time constraints related to thermal machines.

The paper is organized as follows. Section II presents the graph theory fundamentals. It also extends the PPSR approach. Section III presents the graph theory method. Section IV presents the simulation results and the analysis. Section V concludes the paper and presents future work.

\section{POWER SYSTEM RESTORATION AND GRAPH THEORY}

In this section, some graph theory fundamentals, the USC and the $k$-shortest simple paths algorithms are introduced. This section also extends the PPSR approach and details the constraints and the assumptions considered in this paper.

\section{A. Graph Theory Fundamentals}

Multiple power systems properties can be conveniently described by means of a diagram consisting of a set of vertices together with a set of edges joining certain pairs of these vertices. Therefore, an undirected graph $\mathbf{G}=(\mathbf{V}, \mathbf{E}, \mathbf{W})$ is used in this paper to represent an electrical network with $n_{b}$ buses, $n_{g}$ generators and $n_{b s}$ BS units.

In the graph $\mathbf{G}$, the elements $v_{i} \in \mathbf{V}, i=1,2, \ldots, n_{b}$, and $e_{i j} \in \mathbf{E} \subset \mathbf{V} \times \mathbf{V}, i, j=1,2, \ldots, n_{b}$, denote the set of nodes and edges, respectively. The sets $\mathbf{V}$ and $\mathbf{E}$ represent the buses and branches in the power system, respectively. A subset $\mathbf{B} \subset \mathbf{V}$, of size $n_{b s}$, is defined to represent only those generators which are BS units. The elements $w_{i j} \in \mathbf{W}, i, j=1,2, \ldots, n_{b}$, represent the weight factors associated with each edge $e_{i j} \in \mathbf{E}$. The weighted adjacency matrix, denoted as $\mathbf{W}$, is built by using the susceptance in p.u., denoted as $b_{i j}$, between nodes $i$ and $j$. The un-weighted adjacency matrix, denoted as $\mathbf{A}_{\mathbf{W}}$, can also be computed by ignoring the weight factors and considering only the connectivity between nodes $i$ and $j$.

\section{B. Cutset Definition}

In graph theory, a cutset $\mathbf{E}_{\mathbf{S}}$, which partitions $\mathbf{G}$, represents the set of edges that needs to be removed to separate the graph into $r$ sub-graphs. For simplicity, the bisection case $(r=2)$ is initially used to separate $\mathbf{G}$ into two disjoint sub-graphs $\mathbf{G}_{\mathbf{1}}$ and
$\mathbf{G}_{2}$. Nevertheless, by applying recursive bisection [9], [10], the method can also determine the SS for $r>2$. The cut (1) is represented as the sum of the weights of the edges contained in the cutset [11].

$$
\operatorname{cut}\left(\mathbf{V}_{\mathbf{1}}, \mathbf{V}_{\mathbf{2}}\right)=\sum_{e_{i j} \in \mathbf{E}_{\mathrm{S}}} w_{i j}
$$

The graph-cut problem is then defined as finding the cutset that bisects the graph with minimum cut [11] i.e. $\min \left\{\operatorname{cut}\left(\mathbf{V}_{\mathbf{1}}, \mathbf{V}_{\mathbf{2}}\right)\right\}$. In the sectionalizing problem, the set of edges contained in $\mathbf{E}_{\mathbf{S}}$ represents the set of transmission lines in the actual power system that should not be closed to create the islands after the blackout.

\section{Spectral Clustering Algorithm}

Representing the network in blackout as a graph converts the sectionalizing problem into a graph-cut problem. Several methods can be used to solve the graph-cut problem. Spectral clustering is implemented in this paper to determine the SS. The un-normalized Laplacian Matrix, denoted as $\mathbf{L}$, is defined for a graph $\mathbf{G}$ as shown in (2) [11].

$$
\mathbf{L}=\mathbf{D}-\mathbf{W}
$$

In (2), the degree matrix, denoted as $\mathbf{D}$, is defined as the diagonal matrix with the degrees $d_{i}=\sum_{j=1}^{n_{b}} w_{i j}$. The value $d_{i}$ is also equal to the total weight of the edges connected to node $v_{i} \in \mathbf{V}$. Defined in this way, $\mathbf{W}$ and $\mathbf{L}$ are both symmetric for any undirected graph. The USC algorithm, for the bisection case, is summarized as follows [11]:

1. Compute the un-normalized Laplacian Matrix $\mathbf{L}$ (2).

2. Compute the first two eigenvectors $\boldsymbol{\vartheta}_{\mathbf{1}}$ and $\boldsymbol{\vartheta}_{\mathbf{2}}$, corresponding to the two smallest eigenvalues, of the Eigenproblem $\mathbf{L} \vartheta=\lambda \vartheta$.

3. Let $\mathbf{T} \in \mathbf{R}^{n_{b} \times 2}$ be the matrix containing the vectors $\boldsymbol{\vartheta}_{\mathbf{1}}$ and $\boldsymbol{\vartheta}_{2}$ as columns.

4. For $i=1, \ldots, n_{b}$, let $\mathbf{y}_{i} \in \mathbf{R}^{2}$ be the vector corresponding to the $i$-th row of $\mathbf{T}$.

5. Cluster the nodes $\left(\mathbf{y}_{i}\right)_{i=1, \ldots, n_{b}} \in \mathbf{R}^{2}$ into $\mathbf{V}_{\mathbf{1}}$ and $\mathbf{V}_{\mathbf{2}}$ using the $k$-medoids algorithm [12].

When computing the steps mentioned above, the disjoint sets $\mathbf{V}_{\mathbf{1}}$ and $\mathbf{V}_{\mathbf{2}}$ are determined. When requiring more than two islands, the set with various BS units (more than one BS unit) should be used to apply recursive bisection. The previous steps would need to be applied to this new set.

\section{K-Shortest Simple Paths}

Considering two designated nodes $s, t \in \mathbf{V}$, a path $P$ of $\mathbf{G}$ from a BS unit located at node $s$ to a NBS unit or load located at node $t$ is an alternating sequence $v_{0}, e_{0,1}, v_{1}, e_{1,2}, \ldots, e_{n-1, n}, v_{n}$, of distinct nodes $v_{i} \in \mathbf{V}$ and edges $e_{i j} \in \mathbf{E}$, where $v_{0}=s$ and $v_{n}=t$. The total length $l(P)$ from $s$ to $t$ in the path $P$ is defined as the sum of the symmetric lengths $l_{i j}$ contained in $P$. It should be mentioned that the length $l_{i j}$ is defined as one, when computing the number of connections required, or as $1 / w_{i j}$ $\left(w_{i j} \in \mathbf{W}\right)$ when computing the electrical distance between the connected nodes $i$ and $j$. 


$$
l(P)=\sum_{i \in P} l_{i j}
$$

The problem of finding the shortest simple path from $s$ to $t$ is studied by Dijkstra in [13]. However, considering all the problems that might arise during the restoration process, such as sudden unavailability of branches, this paper implements an algorithm to determine the $k$-shortest simple paths [14]. Therefore, when the absolute shortest simple path cannot be implemented during the restoration process, the second or others shortest simple paths can be used instead.

The proposed $k$-shortest simple paths algorithm uses a subroutine of the first simple path to determine the shortest simple path from $s$ to $t$ [13], [14]. Then, a set of $k$-shortest simple paths $\left\{P^{1}, P^{2}, \ldots, P^{k-1}, P^{k}\right\}$ is determined. When $l(P)$ for the $k$ shortest simple paths from the BS unit to all NBS units and loads are computed, the case with minimum number of connections required is selected. In the case when the number of connections between the restored elements to the element to be energized is the same, the shortest electrical distance is used as a second criterion. The algorithm to determine the $k$ shortest simple paths is presented in an ALGOL-like language in [14] and it is illustrated in a flow chart in Fig. 1.

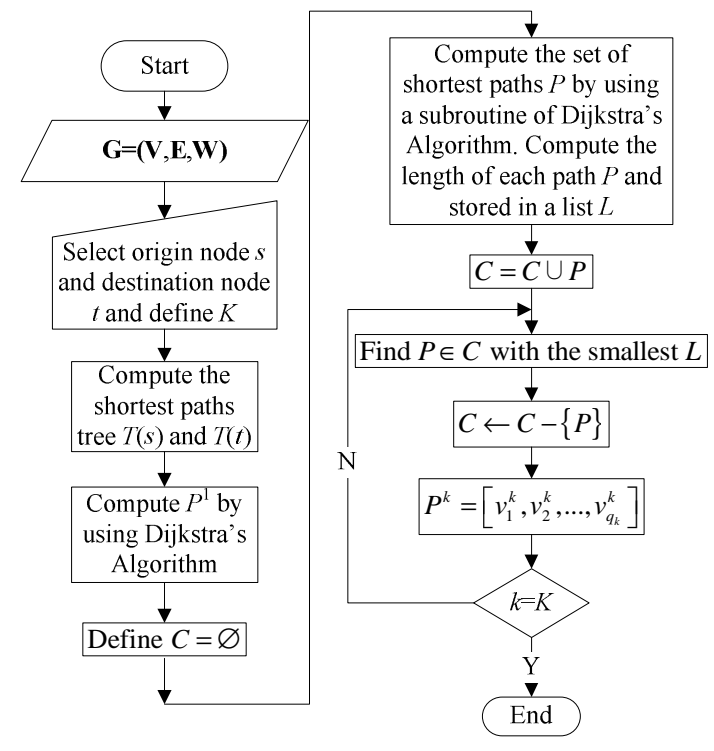

Figure 1. Basic steps for the $k$-shortest simple paths algorithm

\section{E. Parallel Power System Restoration}

When determining the SS to create islands in power systems affected by complete blackouts, several constraints should be taken into account [1]. Typically, the SS must create islands satisfying the following constraints:

i. BS availability and capability within islands,

ii. ability to meet active and reactive power load with the active and reactive power generation in each island,

iii. ability to maintain a suitable voltage profile, to pick up loads, to underexcite generations units, to change taps and to operate synchronous condensers,

iv. the monitoring of each island by the system control center to ensure correct operation, and v. ensuring that all tie-lines are capable of swinging generation and measuring synchronization with adjacent subsystems.

From the above list, the novel graph theory based approach presented in this paper ensures the first, second and fifth constraints. It is assumed that system operators will adequately control the variables in the system during the actual restoration process. It is also considered that the included resources will be used by the operators in an appropriate way to maintain steady-state stability (the frequency and bus voltages within each island) during the restoration process. Transient stability should also be evaluated by simulating the scenarios to restore the islands i.e. by simulating the selected paths to send cranking power to NBS or the paths to pick up loads.

\section{GRAPH TheORY BASED METHOD FOR POWER SyStem RESTORATION}

The proposed graph theory based method is presented in this Section. Fig. 2 shows the flow chart of the proposed method. As noticed, the only input to the method is the graph information presented in Section II-A. Then, the USC algorithm, explained in Section II-C, determines one SS to separate the entire network into islands. The SS creates islands with at least one BS unit and constraints certain branches to be excluded from the SS.

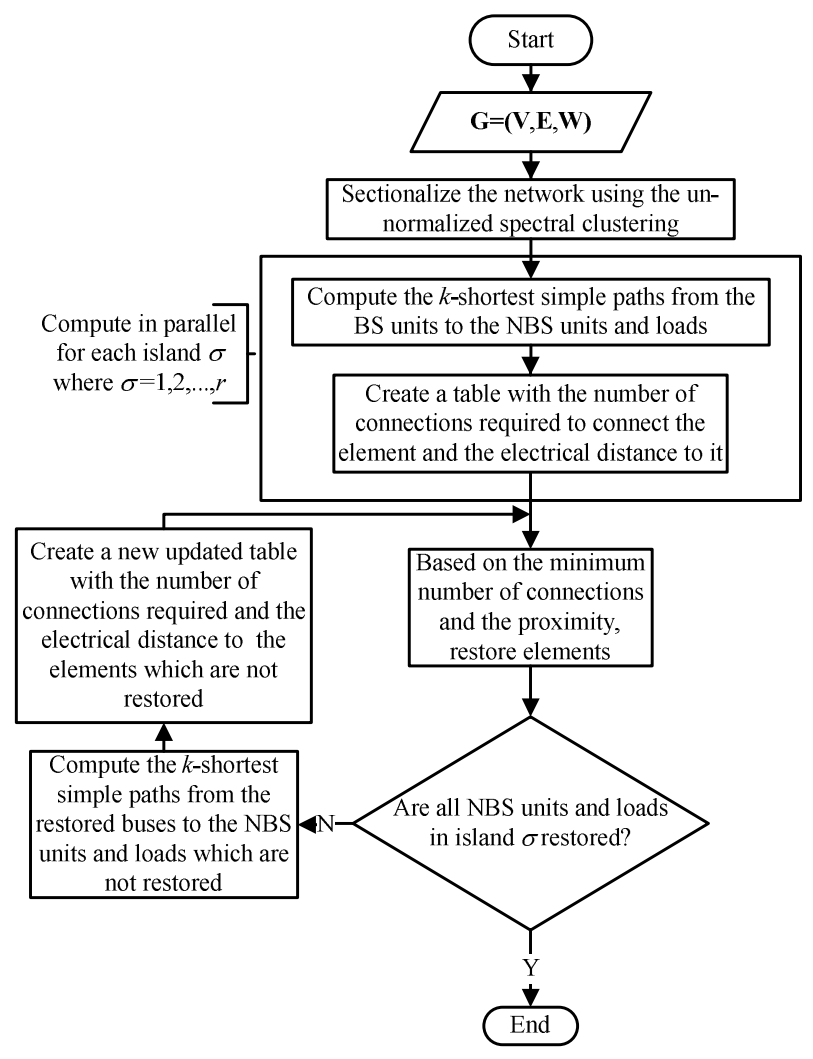

Figure 2. Flow chart of the proposed graph theory based method

To exclude transmission lines without synchro-check, the weight factors associated with the edge, representing the transmission line to be excluded, is changed to a high value. Thus, the modified matrix $\mathbf{W}$ must be computed. To ensure BS availability, the USC algorithm is constrained to include a 
node $v_{i} \in \mathbf{B}$ within each island. When the SS is obtained, the total active power consumption $\left(P_{L, \sigma}^{\max }\right)$ and maximum active power generation $\left(P_{G, \sigma}^{\max }\right)$ within the island $\sigma, \sigma=1,2, \ldots, r$, are computed. Here, it is expected that $P_{L, \sigma}^{\max }$ can be met by $P_{G, \sigma}^{\max }$ within the same island. If the maximum active power generation is smaller than the active power load within the same island, a new SS will be computed. It is important to mention that the final SS must: (i) satisfy the BS availability, (ii) provide sufficient power generation within each island and (iii) ensure that the tie-lines are equipped with synchro-check relays.

When the final SS is determined, the proposed method restores the created islands in parallel. Thus, the restoration process is speeded up. As shown in Fig. 2, the $k$-shortest simple paths from the BS unit in each island (source node $s$ ) to all the NBS units and loads within the same island (destination nodes t) are computed. It should be noted that this process is carried out in parallel for all the created islands. These paths are tabulated with the corresponding values of $l(P)$. When the number of connections and the electrical distance between the BS unit and all the NBS units and loads, within each island, are computed, the element with minimum number of connections is restored. It must be mentioned that CLs, or NBS units, are picked up, or cranked, first. Non-critical loads might be picked up before CLs or NBS units, only if these are in the path from the restored element to the CL to be picked up or to the NBS unit to be cranked. When the number of connections required from the restored element to the element to be energized is the same, the electrical distance is considered. Thus, the electrically closest element is restored first. When this element is restored, a new updated table is computed with the new values of $l(P)$ from the restored buses to the remaining elements which are not restored at this stage of the restoration process. Therefore, the proposed graph theory based method is now converted into an iterative process which updates previous solutions and stops when all elements are restored. This last process is repeated until all NBS units and loads in sub-graph $\mathbf{G}_{\sigma}, \sigma=1,2, \ldots r$, are in service.

\section{Simulation Results}

The proposed graph theory based method for restoring a power system in blackout is tested and validated using the New England 39-bus test system. Fig 3 shows the topology of this test system. The scenario of a complete shutdown is assumed. The susceptance of the lines, the power generation limits and the possible power consumption are obtained from [15]. This paper assumes the availability of three BS units: G3 (node $v_{32}$ ), G4 (node $v_{33}$ ) and G8 (node $v_{37}$ ). Therefore, this test system can be sectionalized into three islands. The remaining generators are considered as NBS units. CLs are located at nodes $v_{7}, v_{18}, v_{21}, v_{23}, v_{26}$. The remaining loads are considered non-critical loads.

The transformers in the New England 39-bus test system are all constrained to be excluded from the SS. The set of edges $\left\{e_{1,2}, e_{3,18}, e_{15,16}\right\}$ is also excluded from possible solutions. These lines are assumed without synchro-check relays. After applying these constraints, the new matrix $\mathbf{W}$ is created. By using the USC algorithm, the solution to the sectionalizing problem is obtained. Since the weight factors associated with the edges represent the susceptance of the branches, long transmission lines are expected to be included in the SS. Therefore, the problems caused by the reconnection of long and unloaded lines during the first stages of the restoration process can be reduced. As a consequence, the solution to the sectionalizing problem reduces high charging currents produced when reconnecting long and unloaded lines during the first stages of PSR.

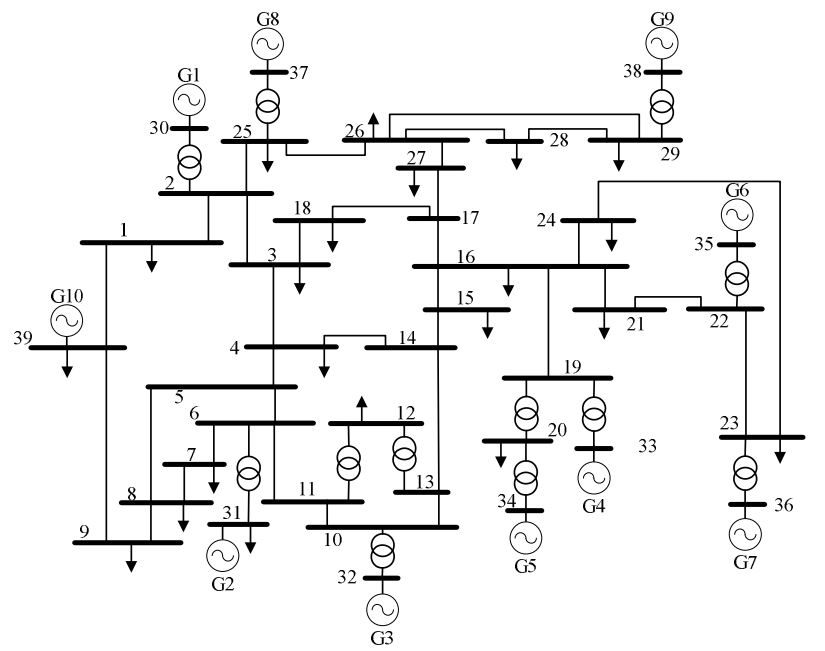

Figure 3. Single line diagram of the New England 39-bus test system

The solution to the sectionalizing problem using the USC algorithm is shown in Fig. 4. As expected, this solution includes a BS unit within each island. Thus, BS units at nodes $v_{37}, v_{32}$ and $v_{33}$ are included in the Island 1, Island 2 and Island 3 , respectively. It can also be noticed that the transformers and lines without synchro-check relays are all excluded from the SS. To conclude that the SS satisfies the considered constraints, the total active power consumption and the maximum active power generation within each island are computed. Table I shows these values for each of the created islands.

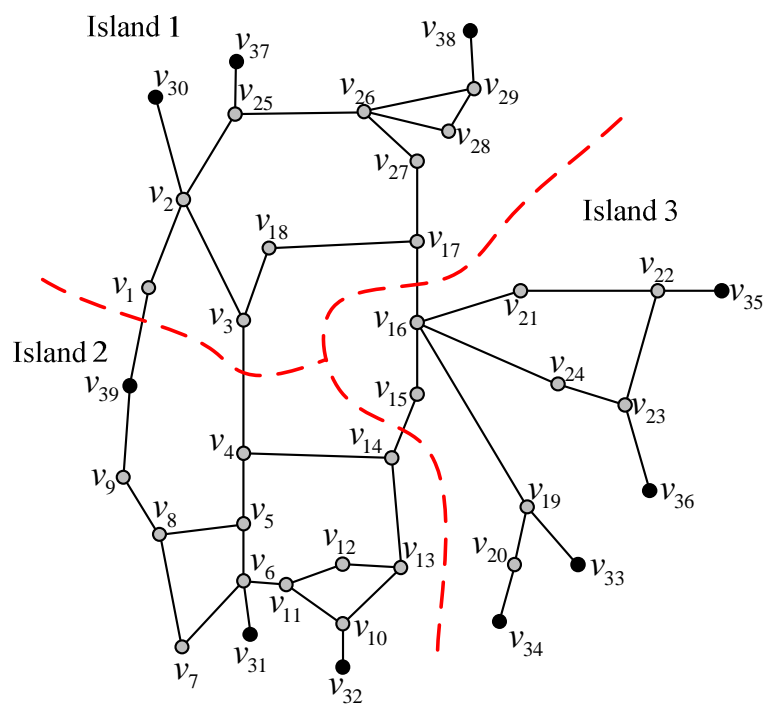

Figure 4. Sectionalizing strategy for the New England 39-bus test system sectionalized into three islands 
As noticed in Table I, there is sufficient active power generation within each island. Therefore, the load consumption within each island can be completely restored. Thus, it is concluded that the created islands are independent. Table I also shows the CLs and the NBS nodes within each island. This information is used to determine the optimal sequence to restore the elements within the islands.

TABLE I. RESULTS WITHIN EACH ISLAND USING THE DETERMINED SS

\begin{tabular}{|c|c|c|c|c|}
\hline $\begin{array}{c}\text { Island } \\
\text { No. }\end{array}$ & $P_{L, \sigma}^{\max }$ & $P_{G, \sigma}^{\max }$ & $\begin{array}{c}\text { Critical Load } \\
\text { Nodes }\end{array}$ & $\begin{array}{c}\text { NBS } \\
\text { nodes }\end{array}$ \\
\hline 1 & 1711.10 & 2469.00 & $v_{18}, v_{26}$ & $v_{30}, v_{38}$ \\
\hline 2 & 2384.03 & 2471.00 & $v_{7}$ & $v_{31}, v_{39}$ \\
\hline 3 & 2159.10 & 2427.00 & $v_{21}, v_{23}$ & $v_{34}, v_{35}, v_{36}$ \\
\hline
\end{tabular}

When the SS is determined, the $k$-shortest simple paths from the source nodes, the BS units, to the destination nodes, the NBS units and loads, are then determined. To evaluate the time required to restore the islands, Table II presents the times to complete restorative actions [6], [16]. These times are considered in the search for transmission paths. Various switching times need to be incorporated when computing the actual restoration time in real power systems [6], [16]. Due to space limitation, the actual restoration of the islands is explained in detail using the Island 1.

TABLE II. TIME TO COMPLETE AN ACTION DURING PSR

\begin{tabular}{|l|c|}
\hline \multicolumn{1}{|c|}{ Action } & Time (min) \\
\hline (A1)-Restart BS unit & 15 \\
\hline (A2)-Energize a bus from BS unit & 5 \\
\hline (A3)-Connect tie line & 25 \\
\hline (A4)-Crank power to a NBS unit from a bus & 15 \\
\hline (A5)-Synchronized subsystems & 20 \\
\hline (A6)-Pick up load & 10 \\
\hline
\end{tabular}

As the BS unit in the Island 1 is located at node $v_{37}$, this node is then considered as the source node. Therefore, the BS unit is in operation after $15 \mathrm{~min}$ (A1 in Table II). For simplicity, the value of $k$ is set to be two. With this value of $k$, path redundancy, defined as multiple paths from $s$ to $t$, is ensured in case of unexpected unavailability of branches. The 2-shortest simple paths to the NBS units and loads in the Island 1 are shown in Table III. The second shortest path is not shown in some cases since the number of connections required is not acceptable for PSR. As noticed in Table III, the load at node $v_{25}$ requires only one connection from the BS unit. Thus, this element is restored after $15 \mathrm{~min}(\mathrm{~A} 2+\mathrm{A} 6)$. Restoring the node $v_{25}$ reduces the number of connections required to restore the $\mathrm{CL}$ at node $v_{26}$. Therefore, as the method focuses on restoring CLs, this load is restored. After $15 \mathrm{~min}(\mathrm{~A} 2+\mathrm{A} 6)$, the CL at node $v_{26}$ is picked up from $v_{25}$. Since the objective is also to restore the NBS units, the NBS unit at node $v_{30}$ is then selected to be restored from $v_{25}$. Thus, after $25 \min (\mathrm{A} 2+\mathrm{A} 2+\mathrm{A} 4)$ this NBS unit is assumed in operation. It must be noted that this paper does not consider the time required by generators to be able to inject power to the system. Also, it should be noted that node $v_{2}$ is a zero injection node.

As it can be noticed, the NBS unit at node $v_{38}$ requires now two connections. This NBS unit is, therefore, restored after 35 min $(\mathrm{A} 2+\mathrm{A} 6+\mathrm{A} 2+\mathrm{A} 4)$. As noticed, when sending cranking power to this NBS unit from node $v_{26}$, the load at node $v_{29}$ is also picked up. Here, it is important to note that if the trans- mission line 26-29 is suddenly unavailable, system operators must use the second shortest path. Thus, it is important to determine various paths in case of unexpected problems during the restoration process.

TABLE III. TwO SHORTEST SIMPLE PATHS TO RESTORE ELEMENTS IN THE ISLAND 1

\begin{tabular}{|c|c|c|c|}
\hline BS unit & $\begin{array}{c}\text { NBS unit/ } \\
\text { Load }\end{array}$ & Path & $\begin{array}{c}\text { Number of } \\
\text { Connections }\end{array}$ \\
\hline$v_{37}$ & $v_{1}$ & $\left\{v_{37}, v_{25}, v_{2}, v_{1}\right\}$ & 3 \\
\hline$v_{37}$ & $v_{3}$ & $\left\{v_{37}, v_{25}, v_{2}, v_{3}\right\}$ & 3 \\
\hline$v_{37}$ & $v_{18}$ & $\left\{v_{37}, v_{25}, v_{2}, v_{3}, v_{18}\right\}$ & 4 \\
\hline$v_{37}$ & $v_{25}$ & $\left\{v_{37}, v_{25}, v_{26}, v_{27}, v_{17}, v_{18}\right\}$ & 5 \\
\hline$v_{37}$ & $v_{26}$ & $\left\{v_{37}, v_{25}\right\}$ & 1 \\
\hline$v_{37}$ & $v_{30}$ & $\left\{v_{37}, v_{25}, v_{26}, v_{30}\right\}$ & 2 \\
\hline$v_{37}$ & $v_{27}$ & $\left\{v_{37}, v_{25}, v_{26}, v_{27}\right\}$ & 3 \\
\hline$v_{37}$ & $v_{28}$ & $\left\{v_{37}, v_{25}, v_{26}, v_{28}\right\}$ & 3 \\
\hline$v_{37}$ & $v_{29}$ & $\left\{v_{37}, v_{25}, v_{26}, v_{29}\right\}$ & 3 \\
\hline$v_{37}$ & $v_{38}$ & $\left\{v_{37}, v_{25}, v_{26}, v_{29}, v_{38}\right\}$ & 4 \\
\hline
\end{tabular}

As it can be noticed, the CL at node $v_{18}$ has not yet been restored. Therefore, the graph theory based method, which focuses on CLs before reconnecting non-CLs, restores this element after $30 \mathrm{~min}(\mathrm{~A} 2+\mathrm{A} 6+\mathrm{A} 2+\mathrm{A} 6)$. This load is picked up from node $v_{2}$, which was previously restored. It should be noted that load at node $v_{3}$ is also restored during this process. To conclude the restoration of the Island 1 , it can be noticed that the load nodes $v_{1}, v_{27}$ and $v_{28}$ are the last three loads to be restored. Considering that these loads cannot be connected at the same time, the loads at nodes $v_{1}, v_{27}$ and $v_{28}$ are restored after $45 \min (\mathrm{A} 2+\mathrm{A} 6+\mathrm{A} 2+\mathrm{A} 6+\mathrm{A} 2+\mathrm{A} 6)$. Nevertheless, it is common that system operators restore various loads at the same time, which will eventually reduce the restoration duration. This Island 1 is completely restored after closing the lines which remain open. As noticed, there are three lines still open $\left(e_{17,18}, e_{17,27}\right.$ and $\left.e_{28,29}\right)$ and these are close after $15 \mathrm{~min}$. Thus, as it can be concluded, the Island 1 is completely restored after 195 min.

Similar analysis can be carried out for the Island 2 and Island 3. Table IV presents the sequence of restoration actions to be carried out in the Island 2. It must be mentioned that the method does not consider the synchronization of zero injections buses as a "Restored Element". In other words, this paper considers the restoration of generation nodes and load nodes with consumption different to zero.

TABLE IV. RESTORATION SEQUENCE FOR THE ISLAND 2

\begin{tabular}{|c|c|c|c|}
\hline Path & $\begin{array}{c}\text { Restored } \\
\text { Elements }\end{array}$ & Actions & $\begin{array}{c}\text { Time } \\
(\text { min })\end{array}$ \\
\hline- & BS unit $v_{32}$ & $\mathrm{~A} 1$ & 15 \\
\hline$\left\{v_{32}, v_{10}, v_{11}, v_{6}, v_{31}\right\}$ & NBS unit $v_{31}$ & $\mathrm{~A} 2+\mathrm{A} 2+\mathrm{A} 2+\mathrm{A} 2+\mathrm{A} 4$ & 35 \\
\hline$\left\{v_{6}, v_{7}\right\}$ & $\mathrm{CL} v_{7}$ & $\mathrm{~A} 2+\mathrm{A} 6$ & 15 \\
\hline$\left\{v_{7}, v_{8}, v_{9}, v_{39}\right\}$ & $\begin{array}{c}\text { Load } v_{8}, v_{9}, v_{39} \\
\text { NBS unit } v_{39}\end{array}$ & $\begin{array}{c}\mathrm{A} 2+\mathrm{A} 6+\mathrm{A} 2+\mathrm{A} 6 \\
+\mathrm{A} 2+\mathrm{A} 4+\mathrm{A} 6\end{array}$ & 60 \\
\hline$\left\{v_{11}, v_{12}\right\}$ & Load $v_{12}$ & $\mathrm{~A} 2+\mathrm{A} 6$ & 15 \\
\hline$\left\{v_{6}, v_{5}, v_{4}\right\}$ & Load $v_{4}$ & $\mathrm{~A} 2+\mathrm{A} 2+\mathrm{A} 6$ & 20 \\
\hline
\end{tabular}

Following the sequence shown in Table IV, the remaining lines still open in the Island 2 must be closed. As it can be determined, there are five lines which must be closed to completely restore the Island 2 . Thus, after 25 min more, for a total of $185 \mathrm{~min}$, this island is restored. 
It is important to note that the method does not consider degree of importance to load buses. It also neglects the thermal time presented in generators. Therefore, it is assumed that these machines can be restored when the graph theory based method determines these must be restored.

Table V shows the sequence of restoration actions to completely restore the Island 3 . This sequence, in addition to the reconnection of line 16-24, completely restores the entire Island 3 after 180 minutes.

TABLE V. RESTORATION SEQUENCE FOR THE ISLAND 3

\begin{tabular}{|c|c|c|c|}
\hline Path & $\begin{array}{c}\text { Restored } \\
\text { Elements }\end{array}$ & Actions & $\begin{array}{c}\text { Time } \\
(\text { min })\end{array}$ \\
\hline- & BS unit $v_{33}$ & $\mathrm{~A} 1$ & 15 \\
\hline$\left\{v_{33}, v_{19}, v_{20}, v_{34}\right\}$ & $\begin{array}{c}\text { Load } v_{20} \\
\text { NBS unit } v_{34}\end{array}$ & $\mathrm{~A} 2+\mathrm{A} 2+\mathrm{A} 6+\mathrm{A} 2+\mathrm{A} 4$ & 40 \\
\hline$\left\{v_{19}, v_{16}, v_{21}\right\}$ & $\begin{array}{c}\text { Load } v_{16} \\
\text { CL } v_{21}\end{array}$ & $\mathrm{~A} 2+\mathrm{A} 6+\mathrm{A} 2+\mathrm{A} 6$ & 30 \\
\hline$\left\{v_{21}, v_{22}, v_{35}\right\}$ & $\mathrm{NBS}$ unit $v_{35}$ & $\mathrm{~A} 2+\mathrm{A} 2+\mathrm{A} 4$ & 25 \\
\hline$\left\{v_{22}, v_{23}, v_{36}\right\}$ & $\begin{array}{c}\text { CL } v_{23} \\
\text { NBS unit } v_{36}\end{array}$ & $\mathrm{~A} 2+\mathrm{A} 6+\mathrm{A} 2+\mathrm{A} 4$ & 35 \\
\hline$\left\{v_{23}, v_{24}\right\}$ & Load $v_{24}$ & $\mathrm{~A} 2+\mathrm{A} 6$ & 15 \\
\hline$\left\{v_{16}, v_{15}\right\}$ & Load $v_{15}$ & $\mathrm{~A} 2+\mathrm{A} 6$ & 15 \\
\hline
\end{tabular}

When the three islands are completely restored, these can be resynchronized. As noted in the SS, four tie-lines are included in this SS. In addition, using the times provided in Table II, it can be noticed that the three islands are resynchronized after 100 minutes.

\section{CONCLUSION AND FUTURE WORK}

This paper presented a novel graph theory based method for restoring large scale power systems affected by complete blackouts. An un-normalized spectral clustering algorithm was implemented to determine the sectionalizing strategy. Including at least one BS unit within each island and ensuring sufficient active power within the created islands allows the efficient and independent restoration in parallel of the created islands. The created islands are restored based on a topological analysis. For this purpose, an efficient $k$-shortest simple paths algorithm was implemented. The minimum number of connections and the electrical distance, from the restored elements to the elements to be energized, were the only consideration taken into account to determine the element to be restarted. By using the number of connections and the electrical distance, the proposed method speeds up the restoration. In addition, cranking power can be rapidly sent to non-blackstart units within each island from the blackstart unit in the same island. In addition, loads in each island can also be picked up quickly. Excluding transmission lines without synchro-check relays from the sectionalizing strategy allows the monitoring of the boundary buses during the resynchronization of the created islands during the last stage of the restoration process.

When computing the SS, reactive power support to system operators was not considered. Thus, it is of great interest to introduce such constraint in future studies. Further studies considering degree of importance in loads, i.e. some loads must be restored before others, are also required. Further research also needs to consider the time constraints related to thermal units, the constraints related to capability curves and the constraints related to cranking power capacity to be sent to
NBS units. These constraints must be taken into account to reflect the actual power system restoration. Ramping time in synchronous machines should also be considered to determine the actual restoration duration. Future work must also focus on restarting NBS units and picking up loads to stabilize machines, considering stability constraints. The time required by NBS units to be able to inject power to the system should also be taken into account. Charging currents produced when reconnecting remaining long and unloaded lines must also be studied. All these issues are of great interest to support system operators during the restoration process.

\section{REFERENCES}

[1] M.M. Adibi, et al., "Power system restoration - A task force report," IEEE Trans. Power Syst., vol. 2, no. 2, pp. 271-277, May 1987.

[2] M.M. Adibi and L.H. Fink, "Power system restoration planning," IEEE Trans. Power Syst., vol. 9, no. 1, pp. 22-28, Feb. 1994.

[3] IEEE PES Power System Engineering Committee, "Special considerations in power system restoration," IEEE Trans. Power Syst., vol. 7, no. 4, pp. 1419-1427, Nov. 1992.

[4] L.H. Fink, K.L. Liou, and C.C. Liu, "From generic restoration actions to specific restoration strategies," IEEE Trans. Power Syst., vol. 10, no. 2, pp. 745-751, May 1995.

[5] M.M. Adibi and R.J. Kafka, "Power system restoration issues," IEEE Computer Applications in Power, vol. 4, no. 2, pp. 19-24, Apr. 1991.

[6] W. Sun, C.C. Liu, and L. Zhang, "Optimal Generator Start-Up Strategy for Bulk Power System Restoration," IEEE Trans. Power Syst., vol. 26, no. 3, pp. 1357-1366, Aug. 2011.

[7] X. Gu and H. Zhong, "Optimisation of network reconfiguration based on a two-layer unit-restarting framework for power system restoration," IET Generation, Transmission \& Distribution, vol. 6, no. 7, pp. 693700, Jul. 2012.

[8] Y. Liu and X. Gu, "Skeleton-network reconfiguration based on topological characteristics of scale-free networks and discrete particle swarm optimization," IEEE Trans. Power Syst., vol. 22, no. 3, pp. 1267-1274, Aug. 2007.

[9] J.A. Bondy and U.S.R. Murty, Graph Theory, 2nd ed.: Springer, 2008.

[10] N. Deo, Graph Theory with Applications to Engineering and Computer Science: Prentice-Hall Inc., 1974.

[11] A.v. Luxburg, "A tutorial on spectral clustering," Statistics and Computing, vol. 17, no. 4, pp. 395-416, Dec. 2007.

[12] L. Kaufman and P. J. Rousseeuw, Finding groups in data: an introduction to cluster analysis. New York: Wiley 1990.

[13] E.W. Dijkstra, "A note on two problems in connexion with graphs," Numerische mathematik, vol. 1, pp. 269-271, 1959.

[14] N. Katoh, T. Ibaraki, and H. Mine, "An efficient algorithm for $k$ shortest simple paths," Networks, vol. 12, pp. 411-427, 1982.

[15] R.D. Zimmerman, C.E. Murillo-Sánchez, and R.J. Thomas, "MATPOWER: Steady-state operations, planning, and analysis tools for power systems research and education," IEEE Trans. Power Syst., vol. 26, no. 1, pp. 12-19, Feb. 2011.

[16] K.L. Liou, C.C. Liu, and R.F. Chu, "Tie line utilization during power system restoration," IEEE Trans. Power Syst., vol. 10, no. 1, pp. 192 199, Feb. 1995. 